Research Article

\title{
Development of Iron Sequester Antioxidant Quercetin@Zno Nanoparticles with Photoprotective Effects on UVA-Irradiated HaCaT Cells
}

\author{
Muhammad Farrukh Nisar $\mathbb{D}^{1,},{ }^{1,2,3}$ Maryam Yousaf, ${ }^{4,5}$ Muhammad Saleem, ${ }^{5,6}$ \\ Hamad Khalid, ${ }^{4}$ Kamal Niaz, ${ }^{7}$ Mustansara Yaqub, ${ }^{4}$ Muhammad Yasir Waqas, ${ }^{2}$ \\ Arsalan Ahmed, ${ }^{4}$ Muhammad Abaid-Ullah, ${ }^{8}$ Jinyin Chen $\mathbb{D}^{1,9}$ Chuying Chen ${ }^{10}{ }^{1}$ \\ Kannan R. R. Rengasamy, ${ }^{10}$ and Chunpeng (Craig) Wan ${ }^{1}$
}

${ }^{1}$ Jiangxi Key Laboratory for Post-Harvest Technology and Nondestructive Testing of Fruits \& Vegetables, Collaborative Innovation Center of Post-Harvest Key Technology and Quality Safety of Fruits and Vegetables in Jiangxi Province, College of Agronomy, Jiangxi Agricultural University, Nanchang 330045, China

${ }^{2}$ Department of Physiology and Biochemistry, Cholistan University of Veterinary and Animal Sciences (CUVAS), Bahawalpur, Punjab 63100, Pakistan

${ }^{3}$ Key Laboratory of Crop Physiology, Ecology and Genetic Breeding, Ministry of Education, Jiangxi Agricultural University, Nanchang, 330045 Jiangxi, China

${ }^{4}$ Interdisciplinary Research Centre in Biomedical Materials (IRCBM), COMSATS University Islamabad, Lahore Campus, Lahore 54000, Pakistan

${ }^{5}$ Department of Chemistry, Faculty of Sciences, University of Kotli, Azad Jammu and Kashmir, Pakistan

${ }^{6}$ Institute of Advance Study, Shenzhen University, Nanshan District, Shenzhen, Guangdong 518060, China

${ }^{7}$ Department of Pharmacology and Toxicology, CUVAS, Bahawalpur, Punjab 63100, Pakistan

${ }^{8}$ Department of Life Sciences, Khwaja Fareed University of Engineering \& Information Technology, Rahim Yar Khan, Pakistan

${ }^{9}$ College of Materials and Chemical Engineering, Pingxiang University, Pingxiang 337055, China

${ }^{10}$ Green Biotechnologies Research Centre of Excellence, University of Limpopo, Private Bag X1106,

Polokwane Sovenga 0727, South Africa

Correspondence should be addressed to Jinyin Chen; jinyinchen@126.com and Chunpeng (Craig) Wan; chunpengwan@jxau.edu.cn

Received 13 July 2021; Accepted 12 August 2021; Published 26 August 2021

Academic Editor: Visweswara Rao Pasupuleti

Copyright ( $) 2021$ Muhammad Farrukh Nisar et al. This is an open access article distributed under the Creative Commons Attribution License, which permits unrestricted use, distribution, and reproduction in any medium, provided the original work is properly cited.

Background. Solar ultraviolet radiation A (UVA, 320-400 nm) is a significant risk factor leading to various human skin conditions such as premature aging or photoaging. This condition is enhanced by UVA-mediated iron release from cellular iron proteins affecting huge populations across the globe. Purpose. Quercetin-loaded zinc oxide nanoparticles (quercetin@ZnO NPs) were prepared to examine its cellular iron sequestration ability to prevent the production of reactive oxygen species (ROS) and inflammatory responses in HaCaT cells. Methods. Quercetin@ZnO NPs were synthesized through a homogenous precipitation method, and the functional groups were characterized by Fourier transform infrared (FTIR) spectroscopy, whereas scanning electron microscopy (SEM) described the morphologies of NPs. MTT and qRT-PCR assays were used to examine cell viability and the expression levels of various inflammatory cytokines. The cyclic voltammetry (CV) was employed to evaluate the redox potential of quercetin- $\mathrm{Fe}^{3+} /$ quercetin- $\mathrm{Fe}^{2+}$ complexes. Results. The material characterization results supported the loading of quercetin molecules on ZnO NPs. The $\mathrm{CV}$ and redox potential assays gave Fe-binding capability of quercetin at $0.15 \mathrm{mM}$ and $0.3 \mathrm{mM}$ of $\mathrm{Fe}\left(\mathrm{NO}_{3}\right)_{3}$. Cytotoxicity assays using quercetin@ZnO NPs with human HaCaT cells showed no cytotoxic effects and help regain cell viability loss following UVA $\left(150 \mathrm{~kJ} / \mathrm{m}^{2}\right)$. Conclusion. Quercetin@ZnO NPs showed that efficient quercetin release action 
is UV-controlled, and the released quercetin molecules have excellent antioxidant, anti-inflammatory, and iron sequestration potential. Quercetin@ZnO NPs have superior biocompatibility to provide UVA protection and medication at once for antiphotoaging therapeutics.

\section{Introduction}

Iron plays a major role in various biological processes of the body and is stored in different proteins and/or as free iron in the cell [1]. Huge amounts of iron are scattered throughout the cytosol and in various cellular organelles especially mitochondria, nucleus, and lysosomes [2,3]. The free iron or cellular labile iron pool (LIP) is comprised of redox-active $\mathrm{Fe}^{2+}$ that can potentially damage cell signalling at multiple sites by forming reactive oxygen species (ROS). The irongenerated ROS can damage cellular lipid bilayers, genetic material (DNA and RNAs), and proteins [4]. Many of the extrinsic factors such as ultraviolet radiation A (UVA) and B (UVB), along with environmental Benz(e)acephenanthrylene (BeA) and chrysene, can increase the oxidative stress, damage DNA, and apoptosis in human skin cells $[5,6]$. Similarly, intrinsic factors such as free iron in spite of the essential roles in the body can be toxic if it accumulated in excess following UVA exposure which may lead to various diseases including cancer, ischemia-reperfusion injury in transplantations, and aging-associated neurodegenerative issues [7]. Chronic ROS efflux creates the cellular oxidative stress in human skin. The oxidative stress leads to skin cancer [810] and damages the natural defence system of the skin [9, $11,12]$. ROS adversely deteriorates biomembranes and biomolecules and induces the expression of matrix metalloproteinases (MMPs) [13], which produce damaged or photoaged skin [14].

Plant active compounds efficiently quench these free ROS and neutralize them to reduce cellular components being damaged and reduce disease progression or incident. On the other hand, many phytochemicals directly quenched the free cellular iron $\left(\mathrm{Fe}^{2+}\right)$ and reduce iron-mediated cellular toxicity and help maintain cellular redox homeostasis [15].

Various environmental factors such as ultraviolet radiations (UVRs), heat, smoke, and chemicals contribute to cellular ROS efflux that triggers the expression of inflammations and induces aging or photoaging and carcinogenesis $[12,14,16]$. UVA radiation is the highest (95\%) of all the UVs reaching the biosphere, and it is a significant risk factor for the above-mentioned conditions, polymorphous light eruption (PLE), and sunburns [17].

Quercetin is a well-known dietary flavonoid present in vegetables, fruits, tea, and brewed drinks [18]. Quercetin has potential beneficial effects on disease prevention through antioxidant properties by scavenging ROS [19] and even antitumor activities [20,21]. Quercetin has been reported to be cytoprotective against various ailments and a potent antioxidant [18]. However, hydrophobicity, strong lipophilic nature, and lower bioavailability hinder the achievement of localized treatments using quercetin.

Zinc oxide $(\mathrm{ZnO})$ is a cheap and ingenious metal oxide used in skincare products for UVR shielding and as a drug carrier [22]. Moreover, it is well known that $\mathrm{ZnO}$ nanoparticles
(NPs) could transform between the hydrophobic to hydrophilic states following UVR exposure and the dark period to unload the drugs $[22,23]$. The current study was aimed at utilizing low doses of UVA light (320-400 or $365 \mathrm{~nm}$ ) along with $\mathrm{ZnO}$-loaded quercetin (quercetin@ZnO) molecules to release the drugs. Following UVA exposures, quercetin@ZnO molecules release the drug for simultaneous skin protection and sunblocking by delivering maximum quercetin molecules to the targeted site. Moreover, the quenching of the free iron molecules, redox potential, anti-inflammatory effects of quercetin on the UVA-driven cytokines, and ROS burst in the HaCaT cells shall also be examined. The method applying quercetin@ZnO may be a useful therapeutic against UVArelated skin condition such as premature photoaging and requires further in-depth investigations for more clinical applications of this cotreatment (quercetin@ZnO+UVA) therapy.

\section{Materials and Methods}

2.1. Development of ZnO Nanoparticles (NPs) and Quercetin Encapsulation. The development of ZnO NPs was done following a previously described method [22]. Quercetin (CAS \# 117-39-5) was purchased from Biopurify Phytochemicals Ltd., China (http://www.biopurify.com), and a small amount of quercetin $(0.20 \mathrm{mg})$ was mixed and homogenized in $5 \mu \mathrm{L}$ of acetone and diluted into various mass ratios of quercetin to $\mathrm{ZnO} \mathrm{NPs}$ (quercetin: $\mathrm{ZnO} ; 1: 100,1: 50,1: 10,1: 1$, $10: 1,50: 1$, and $100: 1)$. Deionized water $(1 \mathrm{~mL})$ was added before ultrasonication in high sound waves to evaporate acetone for a total of an hour. The excessive deionized water was removed through centrifugation (12000 rpm) for 10 min to obtain pure quercetin@ZnO NPs pelleted in the bottom, further dried using $\mathrm{N}_{2}$. Now, acetone $(1 \mathrm{~mL})$ was added and dissolved the loaded quercetin@ZnO NPs and was again centrifuged $(12000 \mathrm{rpm})$ for a further $10 \mathrm{~min}$ for sharp separation. The various concentrations of quercetin@ZnO NPs in the acetone were examined using a UV spectrophotometer (Lambda 900, PerkinElmer, USA) in the wavelength range of $200-900 \mathrm{~nm}$, which calculated the adsorption rate (AR) and drug loading capacity (LC) of quercetin on $\mathrm{ZnO}$ NPs in accordance with the standard curve of quercetin in acetone using the following equation

$$
\begin{aligned}
& \mathrm{AR} \%=\frac{W_{\text {in }}}{W_{\text {raw }}} \times 100 \%, \\
& \mathrm{LC} \%=\frac{W_{\text {in }}}{W_{\text {ZnO }}} \times 100 \%,
\end{aligned}
$$

where $W_{\text {in }}$ is the weight of quercetin in acetone, $W_{\text {raw }}$ is the raw weight of loading quercetin, and $W_{\mathrm{ZnO}}$ is the raw weight of $\mathrm{ZnO}$ for loading quercetin. The verification of results was done in three independent tests. 
2.2. Characterization of $\mathrm{ZnO} N P s$ and Quercetin Release Studies. The shape and size of ZnO NPs were studied by scanning electron microscopy (SEM) (SU-70 HITACHI, Japan). The size distribution of NPs was observed at $25^{\circ} \mathrm{C}$ by using dynamic light scattering equipment (DLS, Zetasizer Nano S90, Malvern, UK) [22]. The element composition was examined through energy-dispersive spectroscopy (EDS) (X-MaxN, Oxford Instruments, UK). The infrared spectrum of ZnO NPs and quercetin@ZnO NPs was determined using a Fourier transform infrared spectrometer (FTIR) (Spectrum GX, PerkinElmer, USA).

The quercetin@ZnO NPs were selected having optimal AR value and used for UVA-controlled specific release studies. Then, we scattered the quercetin@ZnO NPs randomly on the glass slides exposed to a UVA light ( $365 \mathrm{~nm}, 12 \mathrm{Watt}$ ) emitter at $37^{\circ} \mathrm{C}$ under dark conditions. A total of 5 experiment groups with triplicate were performed. Then, irradiate the quercetin@ZnO NPs with UV light, and at different times $(1,2,4,6$, and $8 \mathrm{~h})$, each group of quercetin@ZnO was poured off and cleaned using acetone $(1 \mathrm{~mL})$. The discharged quercetin concentration in the acetone from quercetin@ZnO NPs was examined using UV spectrophotometry which depicts its release or unloading behaviour. Meanwhile, quercetin@ZnO NPs with no UV irradiation were taken as controls.

2.3. Cell Culture Conditions and Pretreatment of Cells with Quercetin. Human epidermal keratinocytes (HaCaT) were kindly donated by Professor Julia Li Zhong, Photobiology Laboratory, College of Bioengineering, Chongqing University, China. HaCaT cells were maintained in Dulbecco's modified Eagle's medium (DMEM) culture medium at the cell culture lab, Interdisciplinary Research Center in Biomedical Materials (IRCBM), CUI, Lahore Campus, Pakistan. $\mathrm{HaCaT}$ cells were maintained in high-glucose DMEM (Gibco), having 10\% $(v / v)$ heat-inactivated fetal bovine serum (FBS) media and also containing $50 \mathrm{U} / \mathrm{mL}$ penicillin/streptomycin. Cells were kept under a humidified cell incubator chamber set at $37^{\circ} \mathrm{C}$ adjusted with $5 \% \mathrm{CO}_{2}$. Cells were pretreated with a range of quercetin concentrations $(0,1.0,2.5,5.0,10.0$, and $20.0 \mu \mathrm{M})$ found through cell viability under various drug doses. After finding the optimum concentration $(20 \mu \mathrm{M})$, cells were exposed to quercetin for $12 \mathrm{~h}$ and exposed to UVA, and then, stress-related studies were conducted.

2.4. UVA Source and Dosimetry. For the UVA treatment to the $\mathrm{HaCaT}$ cells, the UVA lamp with a specific waveband of $365 \mathrm{~nm}$ was used. The dosage was calculated by using standard measurement procedures provided with the lamp. An optimum dose range of UVA was set by applying a range of doses $\left(0,25,75,150,300\right.$, and $\left.450 \mathrm{~kJ} / \mathrm{m}^{2}\right)$ where the cell viability was about $75-80 \%$.

2.5. Binding Assay of Ferric Ion $\left(\mathrm{Fe}^{3+}\right)$ with Quercetin. A stoichiometry amount of $\mathrm{Fe}^{3+}$-binding quercetin molecules was measured with a spectrophotometer. An increased absorbance was observed due to the complex formation of $\mathrm{Fe}^{3+}$ with quercetin. Samples containing $0.3 \mathrm{mM}$ quercetin,
$100 \mathrm{mM} \mathrm{KNO}_{3}$, and $0.5 \mathrm{mM} \mathrm{FeNO}_{3}$ in $50 \mathrm{mM}$ (4-(2-hydroxyethyl)-1-piperazine ethane sulphonic acid solution were prepared. Stability constant $(\log k)$ for quercetin $/ \mathrm{Fe}^{3+}$ is measured by a ligand exchange method. After the addition of EDTA (chelating agent), the decrease in absorbance of $\mathrm{Fe}^{3+}$ by quercetin was observed. Each measurement was taken at $25^{\circ} \mathrm{C}$ using a spectrophotometer.

2.6. Measurement of Redox Potential by Cyclic Voltammetry $(\mathrm{CV})$. CV was employed to evaluate the redox potential of quercetin- $\mathrm{Fe}^{3+} /$ quercetin- $\mathrm{Fe}^{2+}$ complexes. Voltammograms were recorded by using the PalmSens electrochemical sensor interface workstation. A three-electrode system was used comprising of glassy carbon as the working electrode ( $3 \mathrm{~mm}$ in diameter), saturated calomel electrode as the reference electrode, and platinum wire as the counter electrode. For electrochemical measurements, a solution of quercetin $(4.0 \mathrm{mM})$ and $\mathrm{Fe}\left(\mathrm{NO}_{3}\right)_{3}(2.0 \mathrm{mM})$ in a $100 \mathrm{mM}$ phosphate buffer solution with $\mathrm{pH} 7.0$ was prepared and purged with nitrogen for about $20 \mathrm{~min}$ to remove any atmospheric oxygen before the experiment.

2.7. Cytotoxicity of Quercetin@ZnO NPs through the MTT Assay. HaCaT cells were placed at a density of $6 \times 10^{3}$ in every well of a 96-well plate for $24 \mathrm{~h}$ and then pretreated with the quercetin for a further $12 \mathrm{~h}$. Following pretreatment with quercetin, $\mathrm{HaCaT}$ cells were washed three times using sterilized phosphate buffer saline (PBS), and then, let them be incubated with DMEM having MTT (MTS, 3582, Promega) for about 2-4h following the instructions of the manufacturer. Cell viability was examined with a microplate reader (Model 680, Bio-Rad, Hercules, CA, USA) adjusted at $490 \mathrm{~nm}$. For quercetin@ZnO NPs, a similar procedure was adopted; i.e., $\mathrm{HaCaT}$ cells were seeded after $24 \mathrm{~h}$ and pretreated with various concentrations of $\mathrm{ZnO}$, indole, quercetin, quercetin@ $\mathrm{ZnO}$, and $\mathrm{ZnO}$ /indole for an additional $12 \mathrm{~h}$, followed by UVA irradiation $\left(150 \mathrm{~kJ} / \mathrm{m}^{2}\right)$. The HaCaT cells were kept for recovery from harmful effects of UVA radiations for about $12 \mathrm{~h}$ in DMEM containing $1 \%(v / v)$ FBS. After $24 \mathrm{~h}$ post-UVA irradiation, the DMEM (10\% FBS) will be changed with fresh $\operatorname{DMEM}(0.5 \%$ FBS $)$ mixed with MTT and allowed to incubate for a further $2 \mathrm{~h}$ following instructions by the manufacturer. A microplate reader gave measurements about the viable $\mathrm{HaCaT}$ cells at $490 \mathrm{~nm}$. The cytotoxic effect and viable cells under various combinations, i.e., $\mathrm{ZnO}$, indole, quercetin, quercetin@ZnO, and $\mathrm{ZnO} /$ indole, were seen, and the percent of viable cells to that of control was taken.

2.8. Measurement of Intracellular ROS. In this study, HaCaT cells $\left(2.5 \times 10^{5}\right)$ were seeded in 24-well plates and set for incubation for about $24 \mathrm{~h}$; the cells were exposed to UVA $\left(150 \mathrm{~kJ} / \mathrm{m}^{2}\right)$ as the UVA-treated group and pretreated with various concentrations of quercetin $(0,1.0,2.5,5.0,10.0$, and $20.0 \mu \mathrm{M})$ to find an optimum concentration for later treatments. These pretreated cells $(12 \mathrm{~h})$ were also exposed to the UVA dose $\left(150 \mathrm{~kJ} / \mathrm{m}^{2}\right)$ to check the level of intracellular ROS production in pretreated cells using the $2^{\prime}, 7^{\prime}$ -dichlorofluorescein diacetate (DCFH-DA) Reactive Oxygen Species Assay Kit. Cells were then washed twice with 
sterilized PBS, and add $10 \mu \mathrm{mol}$ DCFH-DA $($ DCFH - DA : DMEM $=1: 1000)(10 \mathrm{mM}$ assay kit, Beyotime Inc., S-0033) into each well and incubate them at $37^{\circ} \mathrm{C}$ to detect cellular ROS levels. After $20 \mathrm{~min}$ of incubation following the manufacturer's procedure (Beyotime Inc., Shanghai, China), cells were given a wash three times with sterilized PBS. The HaCaT cells were examined, and photographs were taken using a fluorescent microscope (IX-71, Olympus Corp., Tokyo, Japan).

2.9. RNA Extraction, cDNA Synthesis, and Gene Expression Analysis ( $q R T-P C R)$. Total cellular RNA was extracted from sham or treated (UVA/quercetin) cells using the TRIzol reagent (Invitrogen, Carlsbad, CA, USA) according to the instructions provided by the manufacturer and then digested with RNase-free DNase-I (Promega, Madison, WI, USA) to remove genomic DNA. The extracted RNA's purity was quantified as a ratio of OD260/OD280 and measured with a NanoDrop 1000 spectrophotometer (Thermo Scientific, New York, United States), and RNAs were stored at $-80^{\circ} \mathrm{C}$ for later use. The cDNA was prepared from $1 \mu \mathrm{g}$ of the RNA by reverse transcriptase polymerase chain reaction (RT-PCR). According to the manufacturer's instructions, the RNA was reverse transcribed using SuperScript II Reverse Transcriptase and Random Primer Mix (Invitrogen, USA) in $25 \mu \mathrm{L}$ volume.

Total RNA content in cells was isolated from both groups using an RNA extraction kit (BioTeke Corporation, Beijing, China). First-strand cDNA was synthesized using PrimeScript ${ }^{\circledR}$ RT Reagent Kit with gDNA Eraser (Takara, Dalian, China). PCR amplification of the cDNA products $(1 \mu \mathrm{L})$ was performed with the PCR premix (BioTeke, Wuxi, China) and by using the following primer pairs (Table 1). Using a LightCycler apparatus (C1000 Touch, Bio-Rad, Hercules, CA, USA) with the Promega GoTaq ${ }^{\circledR}$ qPCR Master Mix (A6001) for qRT-PCR experiments, gene-specific primers (Table 1) will be used to analyze the expression levels of the gene. The qRT-PCR was performed in a StepOnePlus Real-Time PCR System using the SYBRVR Premix Ex Taq ${ }^{\mathrm{TM}}$ kit (Takara, Dalian, China). The qRT-PCR procedure included a series of cycles of $95^{\circ} \mathrm{C}$ for $10 \mathrm{~s}$ followed by 40 cycles of $95^{\circ} \mathrm{C}$ for $15 \mathrm{~s}, 60^{\circ} \mathrm{C}$ for $15 \mathrm{~s}$, and $72^{\circ} \mathrm{C}$ for $30 \mathrm{~s}$. Every experiment was laid in triplicate to analyze the relative expression level of the specific inflammatory genes. The GAPDH gene fragment, amplified with primers (Table 1), was used as an internal control.

2.10. Statistical Analysis. The data taken for three separate experiments in triplicate for each sample is represented as mean \pm standard deviation (SD). The statistical significance was found by using Student's $t$-test, and $p$ values of $<0.05$ for all relative experiments are set as significant.

\section{Results}

\subsection{Development and Characterization of $\mathrm{ZnO}$ and Quercetin@ZnO NPs}

3.1.1. SEM Analysis of Quercetin-Loaded ZnO Nanoparticles (Quercetin@ZnO NPs). The characterization of the ZnO NPs
TABle 1: Primer sequences for qRT-PCR analyses.

\begin{tabular}{cccc}
\hline \multirow{2}{*}{ No. Gene name } & $\begin{array}{c}5^{\prime} \\
-3^{\prime}\end{array}$ & Primer sequence $5^{\prime}-3^{\prime}$ \\
\hline \multirow{2}{*}{1} & \multirow{2}{*}{ GAPDH } & F & GAAGATGGTGATGGGATTTC \\
& R & GAAGGTGAAGGTCGGAGTC \\
\hline \multirow{2}{*}{2} & \multirow{2}{*}{ IL-1 $\alpha$} & F & TGAGCTCGCCAGTGAAATGA \\
& & R & AACACGCAGGACAGGTACAG \\
\hline \multirow{3}{*}{3} & \multirow{2}{*}{ IL-6 } & F & CTCAATATTAGAGTCTCAACC \\
& & R & CAGAAGGCAACTGGACCGAA \\
\hline \multirow{2}{*}{4} & \multirow{2}{*}{ NF- $\kappa$ B } & F & AACAGAGAGGATTTCGTTTCCG \\
& & R & TTTGACCTGAGGGTAAGACTTCT \\
\hline \multirow{2}{*}{5} & \multirow{2}{*}{ TNF- $\alpha$} & F & CTGGGCAGGTCTACTTTGGG \\
& & R & CTGGAGGCCCCAGTTTGAAT \\
\hline
\end{tabular}

and quercetin@ZnO is illustrated in Figure 1.SEM evaluated the physical appearance of $\mathrm{ZnO}$ NPs, images were taken on different magnifications, and the $\mathrm{ZnO}$ image was scanned at $2 \mu \mathrm{m}$ which showed a flower-like arrangement of $\mathrm{ZnO}$ NPs with clusters of flowers (Figure 1(a)). SEM imaging of quercetin@ZnO NPs was also scanned on different magnifications (Figure 1(b)), and different ratios of $\mathrm{ZnO}$ nanoparticles and quercetin@ZnO were prepared to select a suitable ratio (quercetin: $\mathrm{ZnO}, 1: 10$ ) for the experiments and clearly showed that quercetin is loaded on ZnO NPs encircled by yellow dotted lines. However, loading is prominent (Figure 1(b)). Moreover, quercetin: $\mathrm{ZnO}$ in the ratio of $1: 50$ has much lower loading than $1: 10$; similarly, the $1: 100$ ratio of quercetin: $\mathrm{ZnO}$ has too much low concentration of quercetin due to 100 percent concentration of $\mathrm{ZnO}$ NPs and only 1 percent concentration of quercetin (data not shown). Nevertheless, as quercetin's concentration increased in different quercetin: $\mathrm{ZnO}$ NPs, ZnO's morphologies get changed and were not prominent (data not shown).

3.1.2. FTIR Analysis of ZnO and Quercetin@ZnO NPs. FTIR spectrums were recorded to examine the quercetin encapsulation with $\mathrm{ZnO}$ NPs (Figure 1(c)). Different concentrations of $\mathrm{ZnO}$ : quercetin NPs were taken, i.e., (a) $100: 1$, (b) 50:1, and (c) $10: 1$, which exhibited a peak at $1500 \mathrm{~cm}^{-1}$ to $1600 \mathrm{~cm}^{-1}$ due to $\mathrm{C}=\mathrm{O}$ stretching and at $3200 \mathrm{~cm}^{-1}$ to $3500 \mathrm{~cm}^{-1}$ due to $\mathrm{OH}$ stretching. These peaks showed a high concentration of $\mathrm{ZnO}$ NPs and low quercetin concentration, but the peaks indicated both functional groups of $\mathrm{ZnO}$ and quercetin. Nevertheless, in $\mathrm{ZnO}$ :quercetin-(d) $1: 1$, the equal concentration of $\mathrm{ZnO} \mathrm{NPs}$ and quercetin showed an absorbance peak at $1400 \mathrm{~cm}^{-1}$ to $1600 \mathrm{~cm}^{-1}$ due to cyclobenzene stretching in quercetin and a peak at $1500 \mathrm{~cm}^{-1}$ to $1600 \mathrm{~cm}^{-1}$ due to carbonyl in both quercetin and $\mathrm{ZnO}$, while the peak at $3200 \mathrm{~cm}^{-1}$ to $3500 \mathrm{~cm}^{-1}$ was due to $\mathrm{OH}$ stretching (Figure $1(\mathrm{c})$ ).

Similar peaks appeared when the concentration of quercetin was increased, i.e., $\mathrm{ZnO}:$ quercetin-(e) $1: 10$, (f) $1: 50$, and (g) $1: 100$, but with great intensity mainly because of the high concentration of quercetin (Figure 1(c)). These results showed successful encapsulation of quercetin with $\mathrm{ZnO}$ 


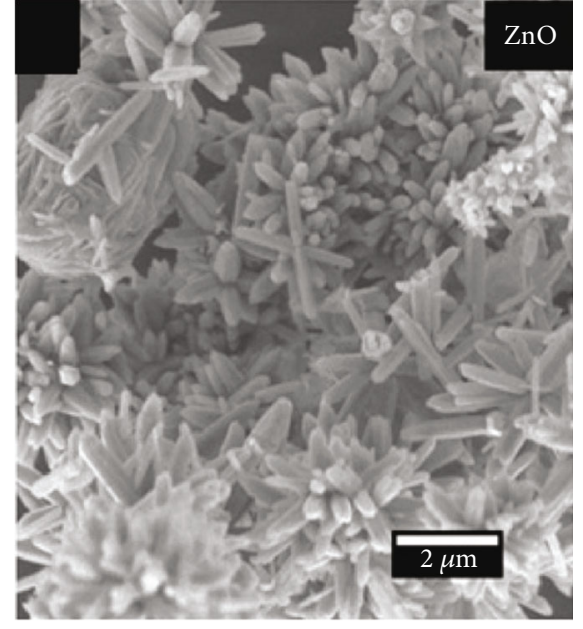

(a)

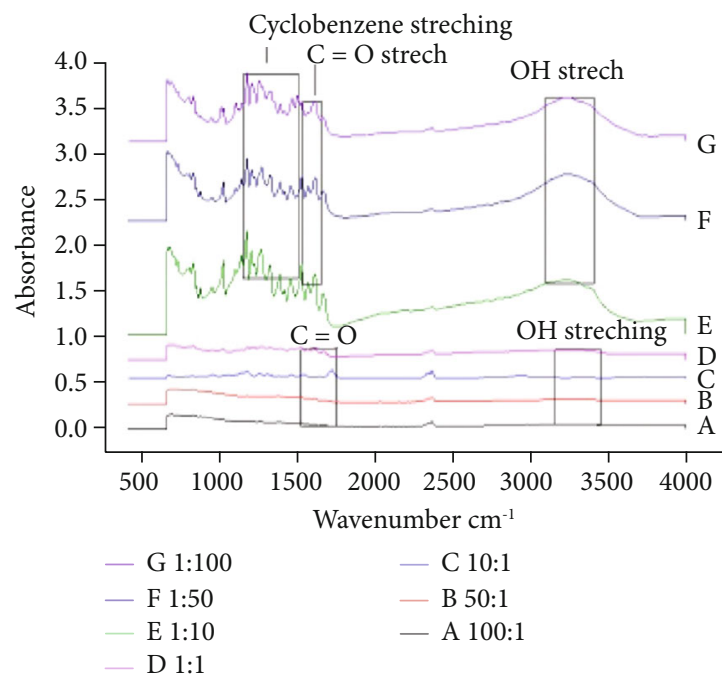

(c)

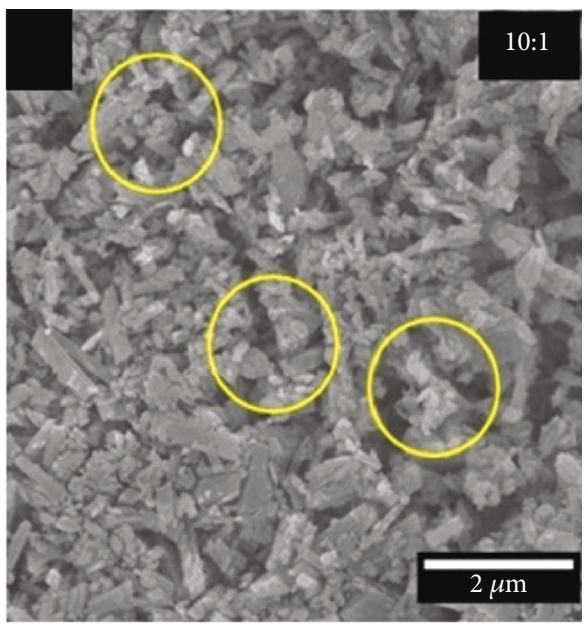

(b)<smiles>O=c1c(O)c(-c2ccc(O)c(O)c2)oc2cc(O)cc(O)c12</smiles>

(d)

FIGURE 1: SEM images of (a) ZnO and (b) quercetin@ZnO nanoparticles (10:1). (c) FTIR results of quercetin@ZnO nanoparticles ( $\mathrm{ZnO}:$ quercetin): a $-100: 1, \mathrm{~b}-50: 1, \mathrm{c}-10: 1, \mathrm{~d}-1: 1, \mathrm{e}-1: 10, \mathrm{f}-1: 50$, and $\mathrm{g}-1: 100$. (d) Chemical structure of quercetin.

NPs to form quercetin@ZnO. The complete structure of quercetin showed multiple bonding sites (Figure 1(d)).

\subsection{Drug Loading and Release}

3.2.1. Adsorption Rate (AR) and Drug Loading Capacity (LC) of $\mathrm{ZnO} N P s$. The reliance on such nanocarriers loaded with drug molecules is purely based on the AR and LC to mass ratio and herein was determined for both $\mathrm{ZnO}$ NPs to quercetin (Figures 2(a) and 2(b)). The highest AR could run up to $99.09 \pm 0.69 \%$ (Figure $2(\mathrm{a})$ ), when the mass ratio of quercetin : $\mathrm{ZnO}$ was 1:100; the LC was unsatisfactory (Figure 2(b)). Overall, the mass ratio of $10: 1$ (quercetin: $\mathrm{ZnO}$ ) received an optimal AR of $90.61 \pm 0.59 \%$ (Figure 2(a)) and LC of 29.35 $\pm 1.62 \%$ (Figure $2(\mathrm{~b})$ ). We were delighted that our analogously round and monodisperse $\mathrm{ZnO}$ NPs could get an optimal AR of $90.61 \pm 0.59 \%$ and LC of $29.35 \pm 1.62 \%$; hence, maximum penetration capability can be achieved as previously reported $[22,24,25]$.
3.3. UV-Controlled Drug Release Behaviour of Quercetin@ZnO. It is aimed at developing UV-controlled release of drugs which is the main concept of this article using quercetin@ZnO NPs to report a potential source for the antiphotoaging effect by adding an iron quenching antioxidant into the skincare formulations. Keeping this in view, concerning quercetin's strong iron chelation capability, this study was designed to evaluate the UV-driven release of the drug from $\mathrm{ZnO}$ NPs (Figure 2(c)). At $8 \mathrm{~h}$ of UVA exposure $\left(150 \mathrm{~kJ} / \mathrm{m}^{2}\right)$, the collective release percent of quercetin was up to $88.71 \pm 3.29 \%$, while $25 \mathrm{~kJ} / \mathrm{m}^{2}$ and $75 \mathrm{~kJ} / \mathrm{m}^{2}$ UVA doses showed $55.29 \pm 1.84 \%$ and $75.71 \pm 2.60 \%$ quercetin release, respectively (Figure $2(\mathrm{c})$ ). On the contrary, the quercetin@ZnO NPs were not exposed to UVA radiation, and only $10.29 \pm 1.15 \%$ of quercetin was released at $8 \mathrm{~h}$ while keeping this in the dark (Figure 2(c)); hence, it proves that the UVA irradiation has some stimulating effects mainly because the UV light creates hydrophobic/hydrophilic transitions in ZnO NPs $[22,23]$. 


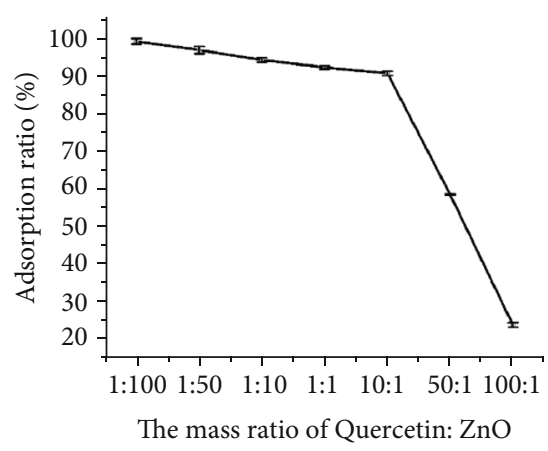

(a)

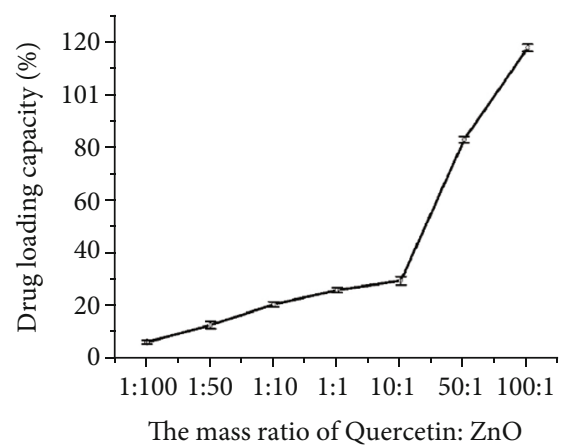

(b)

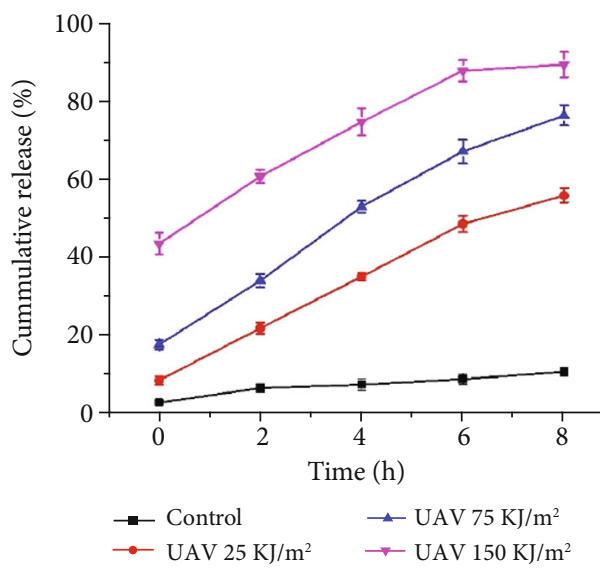

(c)

FIgURE 2: (a) The reliance on percent adsorption rate (AR) of quercetin on $\mathrm{ZnO}$ NPs on the mass ratio of quercetin to $\mathrm{ZnO}$. (b) The reliance on loading capacity (LC) of quercetin on ZnO NPs on quercetin's mass ratio to ZnO. (c) Quercetin's UV-controlled release behaviour for various time points under control, 2, 4, 6, and $8 \mathrm{~h}$ following UVA exposure.

3.4. Measurement of Redox Potential and Iron-Binding Capability of Quercetin. The cyclic voltammogram (CV) of the quercetin- $\mathrm{Fe}^{3+} /$ quercetin- $\mathrm{Fe}^{2+}$ complex exhibited a reversible redox process with a cathodic peak potential EPc of $0.19 \mathrm{~V}$ and an anodic peak potential EPa of $0.09 \mathrm{~V}$ versus saturated calomel electrode (Figure 3(a)). From these two values, the E1/2 was calculated to be $0.14 \mathrm{~V}$ versus a saturated calomel electrode, which corresponds to $0.38 \mathrm{~V}$ versus NHE (normal hydrogen electrode). The iron-binding capability of quercetin was examined by spectrophotometry (Figure 3(b)). Here, we used different concentrations $(0.15 \mathrm{mM}, 0.30 \mathrm{mM})$ of iron nitrate $\left(\mathrm{Fe}\left(\mathrm{NO}_{3}\right)_{3}\right)$ and treated it with quercetin. In the ligand exchange reaction, the absorbance decline at $438 \mathrm{~nm}$ showed an absolute elimination of $\mathrm{Fe}^{3+}$ by EDTA from the quercetin- $\mathrm{Fe}^{3+}$ complex (Figure 3(b)). It is a clear indication of absorbance due to the capturing of $\mathrm{Fe}^{3+}$ by quercetin at $0.15 \mathrm{mM}$ iron nitrate (Figure 3(b)). These results showed that quercetin could reduce ROS and can prevent UV damage. Similar experiments for higher concentrations $(0.30 \mathrm{mM})$ of $\mathrm{Fe}\left(\mathrm{NO}_{3}\right)_{3}$ were also performed (Figure $3(\mathrm{~b})$ ), which gave similar results and where quercetin showed decreased absorbance at $438 \mathrm{~nm}$.

3.5. ROS and Anti-Inflammatory Potential. Quercetin@ZnO NPs are not involved in the reflection or absorption of UVA by the $\mathrm{ZnO}$; instead, they may dissipate the radioactive energies by releasing the quercetin from the $\mathrm{ZnO}$ molecules. UVA is known to create its damaging effects on the skin mainly by the generation of oxidative stress by ROS, which interacts with various intracellular components and specifically the lipid membranes. Quercetin is a natural phytochemical sourced in a considerable number of fruits and vegetables and is reported to reduce oxidative stress and diminish the damage incurred by ROS. In the current study, when the HaCaT cells were exposed to UVA $\left(150 \mathrm{~kJ} / \mathrm{m}^{2}\right)$, which causes the generation of ROS that gave higher fluorescence when stained with dihydroethidium (Figure 4(a)), indicating the production of the high amount of intracellular ROS levels, and when the HaCaT cells were pretreated with various indicated quercetin concentrations (Figure 4(a)), the relative intensity of fluorescence caused by ROS was significantly reduced even up to normal sham cells (Figure 4(a)). The UVA-mediated ROS generation was lowered as pretreated quercetin's concentration gradually increased by neutralizing the ROS efflux in HaCaT cells (Figure 4(a)).

Reducing the expression of inflammatory cytokines may help reduce or slow down the photoaging process in skin cells. Herein, we examined major inflammatory factors' expression playing a decisive role in the onset of photoaging following UVA exposures (Figure 4(b)). The expression levels of various inflammatory factors, viz., IL-1 $\beta$, IL-6, 


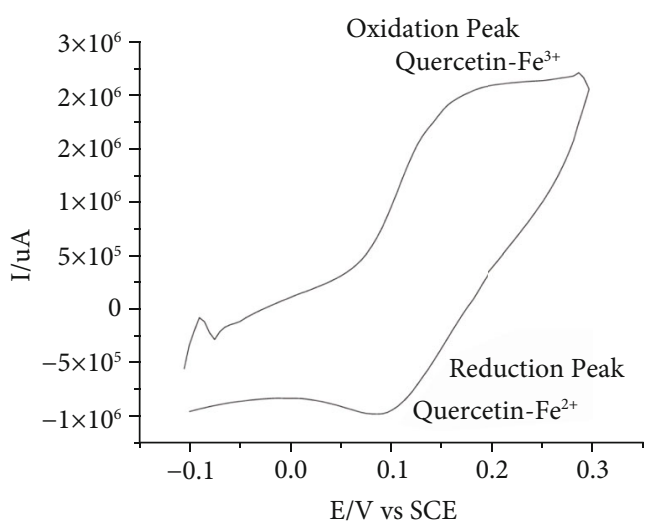

(a)

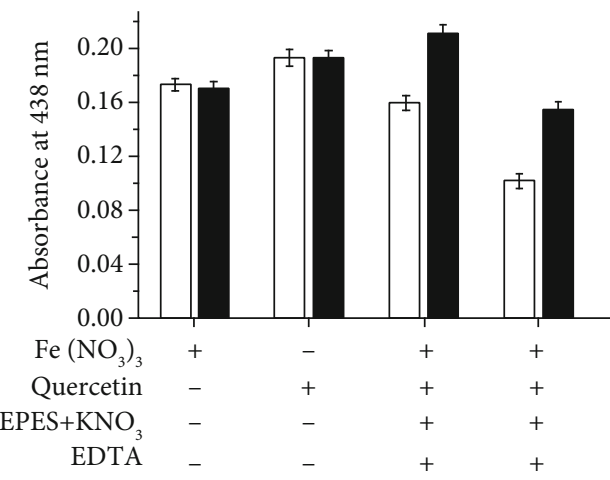

$0.30 \mathrm{mM}$
$0.15 \mathrm{mM}$

(b)

FIGURE 3: Cyclic voltammogram of quercetin-Fe complex $(2: 1)$ in phosphate buffer solution pH 7.0 at a scan rate of $0.1 \mathrm{~V}$ (a). Iron binding of the quercetin- $\mathrm{Fe}^{3+}$ complex with $0.15 \mathrm{mM}$ and $0.30 \mathrm{mM}$ of $\mathrm{Fe}\left(\mathrm{NO}_{3}\right)_{3}$ at $430 \mathrm{~nm}$, which is the $\lambda_{\max }$ of the complex (b).

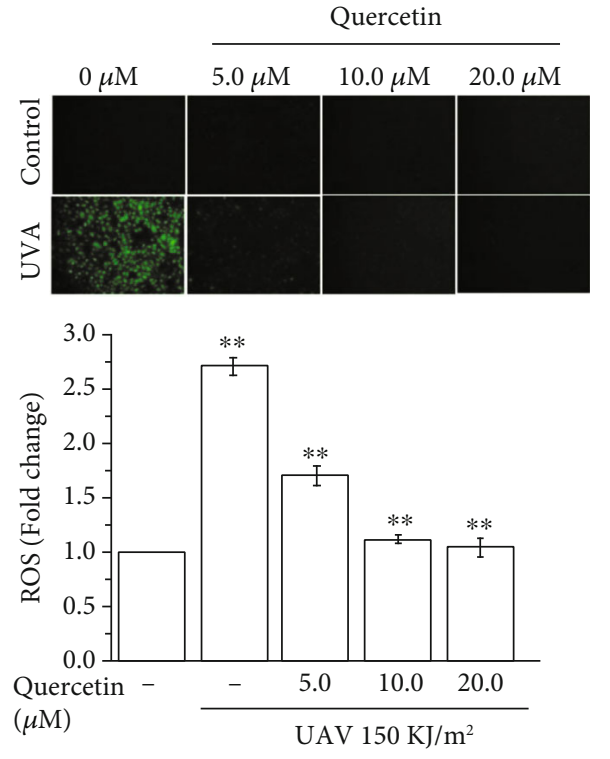

(a)
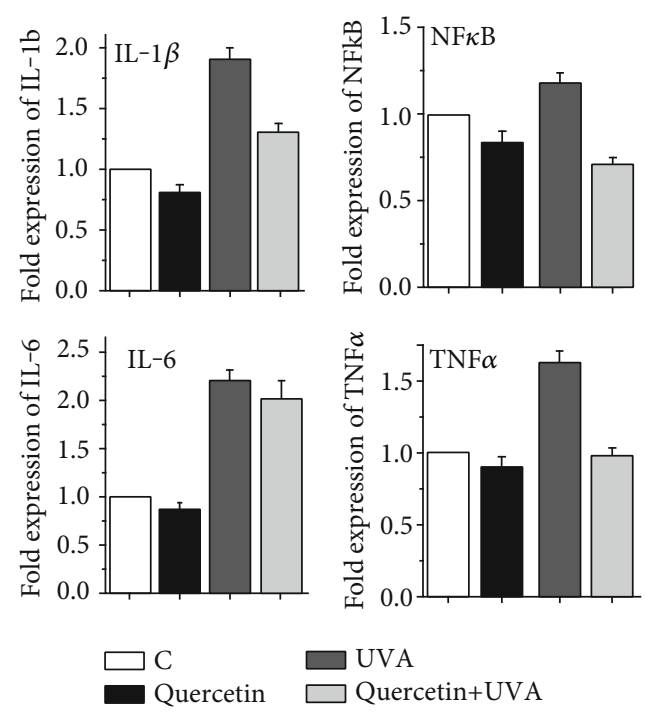

UVA

Quercetin+UVA

(b)

Figure 4: Quercetin@ZnO NPs modulate UVA-induced oxidative stress examined by the relative intensity of fluorescence (a) and inflammatory response (b) in $\mathrm{HaCaT}$ cells $\left({ }^{*} p<0.05,{ }^{* *} p<0.01\right)$.

NF- $\kappa \mathrm{B}$, and TNF- $\alpha$, were also studied in HaCaT cells through qRT-PCR. Quercetin@ZnO NPs showed a significant reduction in UVA-mediated expression of the IL- $1 \beta$ level from 1.9-fold to 1.3-fold, reduce the level of IL-6 from 2.2 -fold to 1.8-fold and the level of NF- $\kappa$ B from 1.18-fold to 0.71 -fold, and reduce the levels of TNF- $\alpha$ from 1.63-fold to 0.93 -fold (Figure 4(b)). The reduction in the level of inflammatory factors indicated a strong effect of quercetin@ZnO NPs on reducing inflammations in $\mathrm{HaCaT}$ cells.

3.6. Cytotoxicity of Quercetin and Biocompatibility. The cytotoxicity assay assessed the use of quercetin@ZnO NPs against UVA for skin protection using $\mathrm{HaCaT}$ cells (Figure 5). Biocompatibility is the main criterion for utilizing biomaterials in clinical applications. Figure 5(a) shows the cell viability loss upon exposure to various UVA doses, but approximately $80 \%$ cell viability was achieved at $150 \mathrm{~kJ} / \mathrm{m}^{2}$, which is considered the optimum dose and used in all experiments herein and as reported in the literature [26]. Herein, increasing the UVA dose decreases the viable cells at $24 \mathrm{~h}$ of postirradiation (Figure 5(a)). The effect of quercetin@ZnO NPs on UVA-induced cytotoxicity in $\mathrm{HaCaT}$ cells was also checked at 8 and $24 \mathrm{~h}$, and no cytotoxicity on the growth of $\mathrm{HaCaT}$ was seen (Figure 5(b)). 


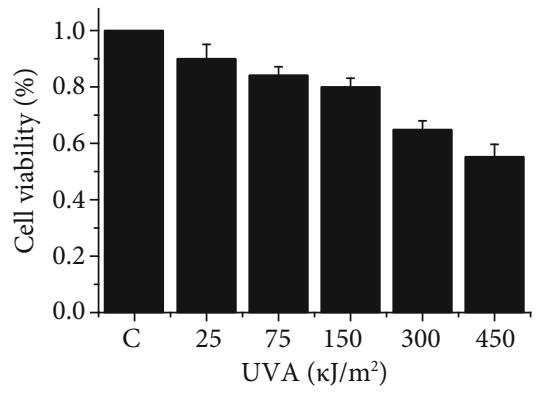

(a)

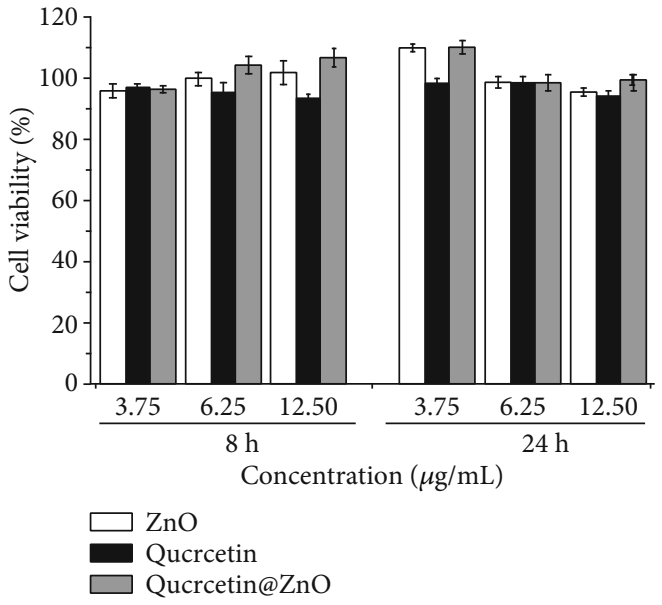

(b)

FIgURE 5: Quercetin's effect on UVA-induced cytotoxicity (a) in human keratinocytes HaCaT at 8 and $24 \mathrm{~h}$ (b).

Moreover, quercetin@ZnO NPs in various ratios and concentrations were applied to HaCaT cells, and all concentrations had no harmful effect and cells showed proliferative behaviour (Figure 5(b)).

\section{Discussion}

UVs contribute to photoaging which is sought mainly due to UVA-mediated iron release in the cells, which creates efflux of ROS, and through various cellular signalling cascade degradation of the ECM and wrinkled skin [17]. ROS creates cellular oxidative stress in human skin leading to multiple issues in skin tissue [8-10] by direct or indirect damage of the natural defense system of the skin which ultimately responds to these stressors $[9,12]$. Quercetin is well known for its ROS-scavenging activities and is more active than many known antioxidants like vitamin E. Quercetin being the dietary flavonoid source in multiple foods (vegetables, fruits, tea, brewed drinks, etc.) has potential beneficial health effects mainly because of its potent antioxidant nature [19]. Besides $\mathrm{ZnO}$, quercetin is also encapsulated with chitosa$\mathrm{n}$ /alginate, where it has shown effective therapeutic potential against oxidative stress-induced liver injury [27].

Primarily, the current study was aimed at demonstrating the use of quercetin@ZnO NPs, designed for potential application as antiphotoaging mainly by quenching the UVAmediated free cellular iron to provide cytoprotection at the same time. Human skin $\mathrm{HaCaT}$ cells play a major role in the progression of various skin diseases and particularly premature photoaging, and hence, the quercetin@ZnO NPs were applied on $\mathrm{HaCaT}$ cells to elucidate their therapeutic potential. Different studies reported the use of various antioxidants providing cytoprotection which have been encapsulated onto either the $\mathrm{ZnO} \mathrm{NPs}$ or chitosan, which are well-known drug carriers $[22,28]$. Using biocompatible materials liable to any particular physical incitement or following any stimuli which may undergo a hydrolytic cleavage, protonation or (supra) molecular conformational changes to release the attached drug are sought to deliver drug molecules at specified sites [29, 30]. UV light/darkdriven consecutive cycles produced an on-demand drug delivery system which is biologically compatible and efficient for shielding skin cells/tissue from UV light which was built up with $\mathrm{ZnO}[22,23]$. Moreover, $\mathrm{ZnO}$ NPs have got strong stability, biocompatibility, and cost-effectiveness but are widely used in drug delivery systems for long [31]. Multiple structural isomorphs have been reported in the literature such as mesoporous $\mathrm{ZnO}$, which can uphold doxorubicin in nanoscale assemblies via adsorption and be released following a slight change in $\mathrm{pH}$ or ultrasound waves [32].

Iron is one of the frequent but essential trace elements in the human body, and both $\mathrm{Fe}^{2+}$ and $\mathrm{Fe}^{3+}$ are reported to take part in different essential physiological processes in almost all eukaryotic cells [33-35]. Due to its widespread nature, iron may quickly donate or accept electrons by engaging in single-electron exchange reactions and potentially producing or amplifying intracellular ROS [36]. These iron-generated ROS can damage cellular lipid layers, nucleic acids, and proteins [4]. Despite the essential roles of iron in the body, it can be toxic if it accumulated in excess and lead to various diseases including cancer, ischemia-reperfusion injury in transplantations, and aging-associated neurodegenerative issues [7]. Furthermore, free cellular iron may induce various types of cutaneous damage by disrupting cellular membranes to cause lipid peroxidation and denaturation of the extracellular matrix leading to premature photoaging [37]. Keeping in view the cutaneous damaging effects of free cellular iron, it is stressed to explore efficient iron sequesters and use skincare products to avoid or slow down the premature photoaging [37]. Quercetin is said to be a potent inhibitor of iron absorption and influence acute and chronic intestinal absorption of iron either by chelation, regulating ferroportin transporter expression inside the intestinal lumen using its 3-hydroxyl group, or by directly altering tissue iron distribution [38]. In the current study, quercetin@ZnO NPs were developed to sequester redox iron in 
order to avoid any interpersonal quercetin bioavailability issues. This may reduce premature photoaging caused by chronic exposures to solar UV-mediated iron overloads. This approach needs further in-depth study to explore the cell signalling cascades driven by quercetin's targeted release following UVA exposures.

Attempts have been made to use various drugs to encapsulate $\mathrm{ZnO}$ to cure various skin ailments, and these drugs use multiple cellular signalling pathways. Quercetin is a prominent flavonoid having antioxidant, anti-inflammatory, and anticancer actions all at once [39]. Quercetin helps reduce the inflammatory cytokine burst in dendritic cells (both myeloid and plasmacytoid dendritic cells) by modulating the transcriptional activation of activator protein 1 (AP1), secretory leucoprotease inhibitor (Slpi), and heme oxygenase-1 (Hmox-1) genes [40]. Quercetin showed to be effective against UVB radiation-mediated damage by downregulation of COX-2 in vitro [41]. In another study, quercetin-loaded chitosan NPs were successfully prepared to reduce UVB-induced damage in $\mathrm{HaCaT}$ cells mainly by lowering the inflammatory responses by downregulation of the NF- $\kappa \mathrm{B} / \mathrm{COX}-2$ signalling pathway [42]. Herein, the current study showed that mimicking results for quercetin@ZnO NPs have strong antioxidant potential and reduce the expression of various inflammatory factors, viz., IL-1 $\beta$, IL-6, NF- $\kappa$ B, and TNF- $\alpha$. Quercetin@ZnO seems to have the potential antitumor therapy [43] and hence could be focused to execute this novel approach of using $\mathrm{Fe}$ sequestration, cytoprotection, or apoptotic cell deaths.

In vivo application of quercetin is relatively low due to less hydrophilicity and little or no absorption at all by the cutaneous tissues [44]. Moreover, bioavailability of quercetin is mainly due to considerable variations among different individuals [45]. Hence, new but universal approaches are being made either directly or indirectly to deliver quercetin mainly using $\mathrm{ZnO}$ NPs at the targeted site. $\mathrm{ZnO}$ nanoparticles have shown superiority among related NPs in drug delivery systems. Herein, the UV reflection property of $\mathrm{ZnO}$ is utilized to lessen harmful effects of UVA as $\mathrm{ZnO}$ actively lodges much of the energy-loaded photons and some of them aid in the discharge or release of quercetin molecules correctly. Hence, further protection to the $\mathrm{HaCaT}$ cells even if extreme UVA exposures release ROS burst can be achieved.

\section{Conclusion}

The results led us to conclude that quercetin@ZnO NPs are entirely developed and characterized, showing that efficient quercetin release actions were found under controlled UVA exposure. Quercetin@ZnO NPs not only reflect UVA light but dissipate the energy-rich photons to release quercetin molecules that bear exceptional antioxidant, anti-inflammatory, and iron-sequestrating potential. Keeping in view the superior biocompatibility, quercetin@ZnO NPs can potentially be combined with UVA for simultaneous protection and medication for antiphotoaging therapeutics.

\section{Abbreviations}
AR: $\quad$ Adsorption rate
cDNA: Complimentary DNA
COX-2: Cyclooxygenase 2
CV: $\quad$ Cyclic voltammetry

DCFH-DA: $2^{\prime}, 7^{\prime}$-Dichlorofluorescein diacetate

DMEM: Dulbecco's modified Eagle's medium

DNA: Deoxyribonucleic acid

ECM: $\quad$ Extracellular matrix

EDTA: $\quad$ Ethylenediaminetetraacetic acid

FBS: $\quad$ Fetal bovine serum

$\mathrm{FeNO}_{3}: \quad$ Ferric nitrate

FTIR: $\quad$ Fourier transform infrared spectroscopy

HaCaT: $\quad$ Human epidermal keratinocytes

IL-1 $\beta$ : $\quad$ Interleukin $1 \beta$

IL-6: $\quad$ Interleukin 6

$\mathrm{kJ} / \mathrm{m}^{2}: \quad$ Kilojoule/square meter

$\mathrm{KNO}_{3}: \quad$ Potassium nitrate

LC: $\quad$ Loading capacity

LIP: $\quad$ Labile iron pool

mM: $\quad$ Millimolar

MMPs: $\quad$ Matrix metalloproteinase

NF- $\kappa$ B: $\quad$ Nuclear factor kappa B

NPs: $\quad$ Nanoparticles

OD: $\quad$ Optical density

PBS: $\quad$ Phosphate buffer saline

qRT-PCR: Quantitative real time polymerase chain reaction

RNA: Ribonucleic acid

ROS: $\quad$ Reactive oxygen species

SD: $\quad$ Standard deviation

SEM: $\quad$ Scanning electron microscope

TNF- $\alpha$ : Tumor necrosis factor alpha

UVA: Ultraviolet radiation A

UVB: $\quad$ Ultraviolet radiation B

UVR: $\quad$ Ultraviolet radiations

ZnO: $\quad$ Zinc oxide

$\mu \mathrm{L}: \quad$ Microlitre.

\section{Data Availability}

The original data can be asked from the first and corresponding authors.

\section{Conflicts of Interest}

The authors declared no conflict of interest for the submission and publication of this article.

\section{Authors' Contributions}

Muhammad Farrukh Nisar (MFN) and Maryam Yousaf conducted the experiments. MFN, Muhammad Saleem, Hamad Khalid, Mustansara Yaqub, Arsalan Ahmed, and Chuying Chen extracted the data and drafted the figures. Muhammad Yasir Waqas, Muhammad Abaid-Ullah, Kamal Niazand, and Kannan RR Rengasamy drafted the manuscript. MFN, Jinyin Chen, and Chunpeng (Craig) Wan 
conceptualized this study and reviewed and wrote this article. Muhammad Farrukh Nisar and Maryam Yousaf contributed equally to this work and are to be considered co-first authors.

\section{Acknowledgments}

The acknowledgements go to the Higher Education Commission (HEC), Pakistan, for funding this study under Startup Research Grant Program No. 21 (1819/SRGP/R\&D/HEC/2018). We also thank Prof. Julia Li Zhong (College of Bioengineering, Chongqing University, China) for providing guidance and the immortal $\mathrm{HaCaT}$ cell line for the experimentation.

\section{References}

[1] J. Wang and K. Pantopoulos, "Regulation of cellular iron metabolism," Biochemical Journal Reviews, vol. 434, no. 3, pp. 365-381, 2011.

[2] F. Petrat, H. De Groot, and U. Rauen, "Subcellular distribution of chelatable iron: a laser scanning microscopic study in isolated hepatocytes and liver endothelial cells," Biochemical Journal, vol. 356, no. 1, pp. 61-69, 2001.

[3] U. Rauen, A. Springer, D. Weisheit et al., "Assessment of chelatable mitochondrial iron by using mitochondrion-selective fluorescent iron indicators with different iron-binding affinities," Chembiochem, vol. 8, no. 3, pp. 341-352, 2007.

[4] K. Jomova and M. Valko, "Advances in metal-induced oxidative stress and human disease," Toxicology, vol. 283, no. 2-3, pp. 65-87, 2011.

[5] D. Ali, R. S. Ray, and R. K. Hans, "UVA-induced cyototoxicity and DNA damaging potential of benz (e) acephenanthrylene," Toxicology letters, vol. 199, no. 2, pp. 193-200, 2010.

[6] D. Ali, A. Verma, F. Mujtaba, A. Dwivedi, R. K. Hans, and R. S. Ray, "UVB-induced apoptosis and DNA damaging potential of chrysene via reactive oxygen species in human keratinocytes," Toxicology Letters, vol. 204, no. 2-3, pp. 199-207, 2011.

[7] T. Nakamura, I. Naguro, and H. Ichijo, "Iron homeostasis and iron-regulated ROS in cell death, senescence and human diseases," Biochimica et Biophysica Acta (BBA)-General Subjects, vol. 1863, no. 9, pp. 1398-1409, 2019.

[8] D. R. Bickers and M. Athar, "Oxidative stress in the pathogenesis of skin disease," Investigative Dermatology, vol. 126, no. 12, pp. 2565-2575, 2006.

[9] M. Ichihashi, M. Ueda, A. Budiyanto et al., "UV-induced skin damage," Toxicology, vol. 189, no. 1-2, pp. 21-39, 2003.

[10] D. P. Steenvoorden and G. M. J. Beijersbergen van Henegouwen, "The use of endogenous antioxidants to improve photoprotection," Photochemistry and Photobiology B: Biology, vol. 41, no. 1-2, pp. 1-10, 1997.

[11] C. J. Gomer, A. Ferrario, N. Rucker, S. Wong, and A. S. Lee, "Glucose regulated protein induction and cellular resistance to oxidative stress mediated by porphyrin photosensitization," Cancer Research, vol. 51, no. 24, pp. 6574-6579, 1991.

[12] R. E. Watson, N. K. Gibbs, C. E. Griffiths, and M. J. Sherratt, "Damage to skin extracellular matrix induced by UV exposure," Antioxidants \& Redox Signaling, vol. 21, no. 7, pp. 1063-1077, 2014.

[13] Y. Tu and T. Quan, "Oxidative stress and human skin connective tissue aging," Cosmetics, vol. 3, no. 3, p. 28, 2016.
[14] P. Pittayapruek, J. Meephansan, O. Prapapan, M. Komine, and M. Ohtsuki, "Role of matrix metalloproteinases in photoaging and photocarcinogenesis," International Journal of Molecular Sciences, vol. 17, no. 6, p. 868, 2016.

[15] E. B. Kurutas, "The importance of antioxidants which play the role in cellular response against oxidative/nitrosative stress: current state," Nutrition Journal, vol. 15, no. 1, p. 71, 2015.

[16] K. S. George, W. Elyassaki, Q. Wu, and S. Wu, "The role of cholesterol in UV light B-induced apoptosis $\dagger$," Photochemistry and Photobiology, vol. 88, no. 5, pp. 1191-1197, 2012.

[17] R. M. Tyrrell, "Modulation of gene expression by the oxidative stress generated in human skin cells by UVA radiation and the restoration of redox homeostasis," Photochemical \& Photobiological Sciences, vol. 11, no. 1, pp. 135-147, 2012.

[18] Y. Li, J. Yao, C. Han et al., "Quercetin, inflammation and immunity," Nutrients, vol. 8, no. 3, p. 167, 2016.

[19] H. N. Jnawali, E. Lee, A. Shin, Y. G. Park, and Y. Kim, "Effect of quercetin in the UV-irradiated human keratinocyte HaCaT cells and a model of its binding to 38 MAPK," Bulletin of the Korean Chemical Society, vol. 35, no. 9, pp. 2787-2790, 2014.

[20] A. K. Calgarotto, V. Maso, G. C. F. Junior et al., “Antitumor activities of quercetin and green tea in xenografts of human leukemia HL60 cells," Scientific Reports, vol. 8, no. 1, p. 3459, 2018.

[21] H. Zhang, M. Zhang, L. Yu, Y. Zhao, N. He, and X. Yang, "Antitumor activities of quercetin and quercetin-5 ',8-disulfonate in human colon and breast cancer cell lines," Food and Chemical Toxicology, vol. 50, no. 5, pp. 1589-1599, 2012.

[22] X. Huang, M. F. Nisar, M. Wang et al., "UV-responsive [email protected] nanoparticles potential for polymorphous light eruption protection and therapy," Materials Science and Engineering: $C$, vol. 107, p. 110254, 2020.

[23] X. Huang, X. Wang, S. Wang, J. Yang, L. Zhong, and J. Pan, "UV and dark-triggered repetitive release and encapsulation of benzophenone-3 from biocompatible $\mathrm{ZnO}$ nanoparticles potential for skin protection," Nanoscale, vol. 5, no. 12, pp. 5596-5601, 2013.

[24] S. J. O'Keefe, B. N. Feltis, T. J. Piva, T. W. Turney, and P. F. Wright, "ZnO nanoparticles and organic chemical UV-filters are equally well tolerated by human immune cells," Nanotoxicology, vol. 10, no. 9, pp. 1287-1296, 2016.

[25] Z. Wang, H. Zhang, L. Zhang, J. Yuan, S. Yan, and C. Wang, "Low-temperature synthesis of $\mathrm{ZnO}$ nanoparticles by solidstate pyrolytic reaction," Nanotechnology, vol. 14, 2002.

[26] S. Yang, B. Zhou, W. Xu et al., "Nrf2- and Bach1 may play a role in the modulation of ultraviolet A-induced oxidative stress by acetyl-11-keto- $\beta$-boswellic acid in skin keratinocytes," Skin Pharmacology and Physiology, vol. 30, no. 1, pp. 13-23, 2017.

[27] V. Tzankova, D. Aluani, M. Kondeva-Burdina et al., "Hepatoprotective and antioxidant activity of quercetin loaded chitosa$\mathrm{n}$ /alginate particles in vitro and in vivo in a model of paracetamol-induced toxicity," Biomedicine \& Pharmacotherapy, vol. 92, pp. 569-579, 2017.

[28] Y. Ding, Y. Qiao, M. Wang et al., "Enhanced neuroprotection of acetyl-11-keto- $\beta$-boswellic acid (AKBA)-loaded Ocarboxymethyl chitosan nanoparticles through antioxidant and anti-inflammatory pathways," Molecular Neurobiology, vol. 53, no. 6, pp. 3842-3853, 2016.

[29] M. A. Azagarsamy, D. L. Alge, S. J. Radhakrishnan, M. W. Tibbitt, and K. S. Anseth, "Photocontrolled nanoparticles for ondemand release of proteins," Biomacromolecules, vol. 13, no. 8, pp. 2219-2224, 2012. 
[30] C. B. Rodell, J. E. Mealy, and J. A. Burdick, "Supramolecular guest-host interactions for the preparation of biomedical materials," Bioconjugate Chemistry, vol. 26, no. 12, pp. 22792289, 2015.

[31] J. Jiang, J. Pi, and J. Cai, "The advancing of zinc oxide nanoparticles for biomedical applications," Bioinorganic Chemistry and Applications, vol. 2018, Article ID 1062562, 18 pages, 2018.

[32] K. Barick, S. Nigam, and D. Bahadur, "Nanoscale assembly of mesoporous ZnO: a potential drug carrier," Materials Chemistry, vol. 20, no. 31, pp. 6446-6452, 2010.

[33] M. W. Hentze, M. U. Muckenthaler, and N. C. Andrews, "Balancing acts: molecular control of mammalian iron metabolism," Cell, vol. 117, no. 3, pp. 285-297, 2004.

[34] D. R. Richardson, "Therapeutic potential of iron chelators in cancer therapy," in Iron chelation therapy, pp. 231-249, Springer, 2002.

[35] D. R. Richardson and P. Ponka, "The molecular mechanisms of the metabolism and transport of iron in normal and neoplastic cells," Biochimica Et Biophysica Acta (BBA)-Reviews on Biomembranes, vol. 1331, no. 1, pp. 1-40, 1997.

[36] E. Graf, J. R. Mahoney, R. G. Bryant, and J. W. Eaton, "Ironcatalyzed hydroxyl radical formation. Stringent requirement for free iron coordination site," Biological Chemistry, vol. 259, no. 6, pp. 3620-3624, 1984.

[37] Y. Ishitsuka, M. Kobayashi, K. Arakane et al., "A novel antiphotoaging ingredient with the effect of iron sequestering," Journal of Dermatological Science Supplement, vol. 1, no. 2, pp. S45-S52, 2005.

[38] M. Lesjak, R. Hoque, S. Balesaria et al., "Quercetin inhibits intestinal iron absorption and ferroportin transporter expression in vivo and in vitro," PLoS One, vol. 9, no. 7, article e102900, 2014.

[39] J. Formica and W. Regelson, "Review of the biology of quercetin and related bioflavonoids," Food and Chemical Toxicology, vol. 33, no. 12, pp. 1061-1080, 1995.

[40] G. Verna, M. Liso, E. Cavalcanti et al., "Quercetin administration suppresses the cytokine storm in myeloid and plasmacytoid dendritic cells," International Journal of Molecular Sciences, vol. 22, no. 15, p. 8349, 2021.

[41] P. Steerenberg, J. Garssen, P. Dortant et al., "The effect of oral quercetin on UVB-induced tumor growth and local immunosuppression in SKH-1," Cancer Letters, vol. 114, no. 1-2, pp. 187-189, 1997.

[42] W. Nan, L. Ding, H. Chen et al., "Topical use of quercetinloaded chitosan nanoparticles against ultraviolet B radiation," Frontiers in Pharmacology, vol. 9, p. 826, 2018.

[43] M. Hashemzaei, A. D. Far, A. Yari et al., "Anticancer and apoptosis-inducing effects of quercetin in vitro and in vivo," Oncology Reports, vol. 38, no. 2, pp. 819-828, 2017.

[44] C. F. Hung, C. L. Fang, S. A. Al-Suwayeh, S. Y. Yang, and J. Y. Fang, "Evaluation of drug and sunscreen permeation via skin irradiated with UVA and UVB: comparisons of normal skin and chronologically aged skin," Dermatological Science, vol. 68, no. 3, pp. 135-148, 2012.

[45] A. F. Almeida, G. I. A. Borge, M. Piskula et al., "Bioavailability of quercetin in humans with a focus on interindividual variation," Comprehensive Reviews in Food Science and Food Safety, vol. 17, no. 3, pp. 714-731, 2018. 